\section{Aplicação do protocolo do "Projeto Nascer Maternidades" em uma maternidade de referência em Feira de Santana, Bahia, Brasil}

\author{
Implementation of the "Projeto Nascer \\ Maternidades" in a reference maternity of \\ the city of Feira de Santana, State of Bahia, \\ Brazil
}

Normeide Pedreira dos Santos 1

Bernardo Galvão Castro2

Maria Fernanda Rios-Grassi3

1-3 Pós-Graduação em Medicina e Saúde Humana. Escola Bahiana de Medicina e Saúde Pública. Campus II. Av. Dom João VI, 275. Salvador, Bahia, Brasil. CEP: 40.290-000 E-mail: grassi@bahia.fiocruz.br

\begin{abstract}
Objectives: to analyze the implementation of the Nascer Maternidades Project protocol, a guideline from the Brazilian Ministry of Health for prophylactic/therapeutic measures on delivery for pregnant women whose serological status vis-à-vis syphilis/HIV was unknown in the first year of implementation, at Maternity Hospital Referral Centre in Feira de Santana, Bahia, Brazil.

Methods: the women's serum was analyzed for HIV and syphilis, as requested during prenatal sessions or at the maternity hospital, and prophylactic and/or therapeutic measures were adopted. Data were obtained from 337 randomized medical records of pregnant women admitted to obstetric care between July 2003 and July 2004.

Results: during the prenatal follow-up, $8.4 \%$ and $8.0 \%$ of the pregnant women were tested for HIV and syphilis, respectively. On delivery, HIV rapid test was performed to $17.7 \%$ and VDRL to $97.8 \%$. Prophylactic measures for HIV were taken on delivery in the case of HIV-infected women. Eighty percent of the VDRL positive women were not treated for syphilis, although all their newborns were treated.

Conclusions: the "Nascer Maternidades Project" guidelines were ineffective during the first year of implementation at the maternity hospital under study.
\end{abstract} Key words HIV, Syphilis, Pregnancy, Disease transmission, vertical

\section{Resumo}

Objetivos: analisar a aplicação do protocolo do "Projeto Nascer Maternidades", estratégia do Ministério da Saúde para a adoção de medidas profiláticas elou terapêuticas no momento do parto, para gestantes com sorologias para sifilis/Aids desconhecidas, no primeiro ano de implantação em uma maternidade de referência em Feira de Santana, Bahia, Brasil.

Métodos: foram analisadas sorologias para HIV e sifilis solicitadas no pré-natal e na maternidade e a adoção de medidas profiláticas elou terapêuticas. Os dados foram coletados em uma amostra randomizada de 337 prontuários de gestantes admitidas para assistência obstétrica no período de julho de 2003 a julho de 2004.

Resultados: no pré-natal, 8,4\% das gestantes realizaram sorologia para $\mathrm{HIV}$ e $8 \%$ para sifilis. $\mathrm{Na}$ maternidade, o teste rápido para HIV foi realizado para $17,7 \%$ das gestantes e o VDRL para $97,8 \%$. A profilaxia das gestantes infectadas pelo HIV foi aplicada no momento do parto. Quanto à sífilis, não houve tratamento para $80 \%$ das mulheres com VDRL reagente. Os recém-nascidos foram tratados.

Conclusões: as determinações do "Projeto Nascer-Maternidades" não foram seguidas no primeiro ano de implantação na maternidade estudada.

Palavras-chave HIV, Sifilis, Gravidez, Transmissão vertical de doença 


\section{Introdução}

A Aids pediátrica e a sífilis congênita exercem grande impacto sobre a saúde materno-infantil. A transmissão vertical é a via de aquisição do HIV para 90,6\% de todas as crianças com Aids. No Brasil, cerca de 10.000 crianças menores de 13 anos foram infectadas por transmissão vertical. ${ }^{1} \mathrm{Na}$ Bahia, foram notificados 206 casos de transmissão maternoinfantil do HIV. 2

Quanto à sífilis, a infecção durante a gestação pode resultar em sífilis congênita, manifestada através de aborto, morte fetal ou doença sintomática precoce ou tardia. ${ }^{3}$ No Brasil, a prevalência de sífilis em gestantes foi estimada em 1,6\% (variando de $1,3 \%$ na região Centro-Oeste a $1,9 \%$ no Nordeste), e a taxa de transmissão pode ocorrer em $25 \%$, estimando-se em $12.000^{1}$ o número de nascidos vivos com sífilis congênita. 4

A adoção de medidas profiláticas, baseadas no Protocolo 076 do Aids Clinical Trial Group, o qual demonstrou a redução da transmissão vertical do HIV em cerca de $67 \%$, teve importante impacto. 5 Entretanto, estima-se que 15 a $30 \%$ dos filhos de mulheres infectadas adquirem o HIV por não ter sido realizada a profilaxia. 6 Destacam-se como falhas na aplicação dos protocolos a ausência de solicitação do teste para HIV na gestação e a qualidade do aconselhamento prévio à solicitação do exame, quando essa ocorre. 7,8 No Brasil, a taxa de transmissão vertical de HIV tem reduzido devido à aplicação de medidas profiláticas, passando de 16\% em 19979 a $7,5 \%$, entre 2003 e 2004 , de acordo com estudo multicêntrico. 10

Apesar de o Ministério da Saúde recomendar testagem universal das gestantes no pré-natal para HIV e sífilis, ${ }^{11}$ os dados disponíveis sugerem que essas infecções são sub-diagnosticadas na gestação. A qualidade do pré-natal está implicada na incidência e no desfecho da sífilis congênita, havendo uma relação inversa entre o número de consultas pré-natal e o risco de resultados fatais. ${ }^{12,13}$

O "Projeto Nascer Maternidades" foi instituído em 2002 no Sistema Único de Saúde (SUS), 14 visando resgatar a oportunidade perdida de diagnóstico pré-natal. Suas diretrizes determinam a realização de testes de todas as parturientes que não comprovem, peri-parto, sorologias negativas para HIV e sífilis realizadas no pré-natal e a adoção de medidas profiláticas e/ou terapêuticas, face à positividade dos exames. A triagem diagnóstica é realizada através do teste rápido para HIV e do VDRL.15

Feira de Santana é a segunda cidade do estado da Bahia com maior número de casos acumulados de
Aids (578), até 2004.2 O número de casos notificados de sífilis congênita cresceu entre os anos de 2000 e 2005, passando de 5 a 32, respectivamente, correspondendo, neste último ano, a cerca de 3 casos por 1000 nascidos vivos. Até outubro de 2006, já foram notificados 20 casos, podendo indicar que a transmissão vertical da sífilis ainda não está completamente controlada nesse município. 16 O Projeto Nascer-Maternidades foi implantado em cinco maternidades dessa cidade em junho de 2003, após capacitação das equipes conforme padronização do Ministério da Saúde. 15

O presente trabalho analisa, pela primeira vez, nesse município, a aplicação das normas preconizadas pelo Projeto Nascer-Maternidades em uma das maternidades de referência, no período imediato à implantação desse projeto.

\section{Métodos}

Trata-se de um estudo retrospectivo, descritivo, com o objetivo de verificar a realização de exames sorológicos para HIV e sífilis no pré-natal e na maternidade e intervenções terapêuticas e/ou profiláticas quando indicados.

O estudo foi baseado no período de julho de 2003 a julho de 2004 (no primeiro ano de implantação do "Projeto Nascer-Maternidades"), em uma das maternidades de referência, localizada em Feira de Santana, município com população estimada em 527.625 habitantes. 17 Os autores optaram por manter sigilo quanto ao nome da maternidade, devido ao escopo do trabalho.

A instituição foi escolhida porque, entre as cinco maternidades de referência, foi a que teve a maior proporção de profissionais capacitados para esse projeto: $100 \%$ dos pediatras vinculados à assistência perinatal, 33,3\% dos residentes de pediatria, 33,3\% dos obstetras, $75 \%$ dos residentes de obstetrícia da época, $100 \%$ das enfermeiras e $86,6 \%$ dos técnicos e auxiliares de enfermagem. A capacitação foi realizada pelas Oficinas de Capacitação para o "Projeto Nascer-Maternidades" em Feira de Santana, 2003, a qual posteriormente resultou em um relatório técnico da Coordenação Municipal DST/HIV/AIDS.

As internações para assistência obstétrica (partos ou abortamentos) realizadas no período do estudo (1182) foram identificadas no livro de registros de admissões do Centro Obstétrico. O tamanho da amostra foi estimado em 282 prontuários (alfa=5\%; estimativa da proporção verdadeira de prevenção correta: $60 \%$, para uma imprecisão máxima de 5\%). O tamanho da amostra foi corrigido acrescentando- 
se $10 \%$ (28) prontuários por previsão de perdas e mais $20 \%$ (56) pela não estratificação da população antes da randomização. Foram selecionados randomicamente 366 prontuários.

Os dados foram coletados nos prontuários obstétricos das gestantes envolvidas no estudo, sendo também utilizadas fontes complementares como prontuários dos recém-nascidos, declarações de nascidos vivos e de óbitos, livros de ocorrências do centro obstétrico e do laboratório do hospital. A ausência de informações necessárias ao estudo nas fontes de pesquisa foi considerada critério de exclusão.

As seguintes variáveis maternas foram consideradas de interesse para o estudo: idade, procedência, estado civil, paridade, realização de pré-natal, tipo de parto, via de parto, produto da concepção, realização de testes de triagem para HIV (sorologia no pré-natal, teste rápido na maternidade) e para sífilis (VDRL), uso de terapêutica no pré-natal, adoção de medidas profiláticas e/ou terapêuticas na maternidade face à positividade dos testes. Para os recémnascidos de mães com sorologias positivas para HIV e/ou sífilis foram pesquisados: sexo, peso, idade gestacional, realização de VDRL, medidas terapêuticas adotadas se VDRL reagente, e profilaxia para transmissão vertical do HIV.

Os dados coletados foram expressos em medidas de tendência central (média, mediana, proporções) e de dispersão (desvio padrão). Para as análises estatísticas foi utilizado o software BioEstat versão 3.0 (Sociedade civil Mamimura/MCT-CNPq).

$\mathrm{O}$ estudo foi autorizado pelo diretor da instituição e realizado após aprovação do projeto de pesquisa pelo Comitê de Ética em Pesquisa da Fundação Bahiana para o Desenvolvimento das Ciências (parecer $n^{\circ} 39 / 2005$ ), estando, portanto, condicionado ao cumprimento dos princípios éticos contidos na Declaração de Helsinki (1964, reformulada em 1975, 1983, 1989, 1996 e 2000) da World Medical Association, e na Resolução CNS 196/96.

\section{Resultados}

Da amostra inicial de 366 gestantes, foram analisados os prontuários e registros de 337 destas. Houve perda de 29 (8\%) prontuários, os quais não foram localizados no arquivo. As características demográficas e obstétricas das gestantes estão descritas na Tabela 1: a média de idade por grupos foi $24,5 \pm 6,5$ anos, com mediana de 23 (variando de 14 a 47 anos). O maior número de gestantes (33\%) estava concentrado na faixa etária de 20 a 24 anos. A
Tabela 1

Características gerais das 337 gestantes avaliadas no primeiro ano de implantação do "Projeto Nascer Maternidades" em uma maternidade de referência em Feira de Santana, Bahia.

\begin{tabular}{|c|c|c|}
\hline Variável & $\mathbf{n}$ & $\%$ \\
\hline \multicolumn{3}{|l|}{ Idade (anos) } \\
\hline Média \pm DP & $24,5 \pm 6,5$ & \\
\hline Mediana & $23(14$ a 47$)$ & \\
\hline $14-19$ & 81 & 24,0 \\
\hline $20-24$ & 111 & 33,0 \\
\hline $25-29$ & 73 & 21,6 \\
\hline $30-34$ & 41 & 12,1 \\
\hline $35-39$ & 20 & 6,0 \\
\hline$>40$ & 9 & 2,7 \\
\hline Não informado & 2 & 0,6 \\
\hline \multicolumn{3}{|l|}{ Procedência } \\
\hline Feira de Santana & 208 & 61,7 \\
\hline Outros municípios & 129 & 38,3 \\
\hline \multicolumn{3}{|l|}{ Estado civil } \\
\hline Casada & 99 & 29,4 \\
\hline Solteira & 182 & 54,0 \\
\hline Viúva & 2 & 0,6 \\
\hline Não informado & 54 & 16,0 \\
\hline \multicolumn{3}{|c|}{ Número de gestações (inclusive a atual) } \\
\hline Média \pm DP & $2,5 \pm 2,2$ & \\
\hline Mediana (de 1 a 18) & 2 & \\
\hline Não informado & 10 & \\
\hline \multicolumn{3}{|l|}{ Via de parto* } \\
\hline Vaginal & 215 & 63,8 \\
\hline Cesárea & 122 & 36,2 \\
\hline \multicolumn{3}{|l|}{ Tipo de parto* } \\
\hline Simples & 329 & 97,6 \\
\hline Múltiplo (duplo) & 8 & 2,4 \\
\hline \multicolumn{3}{|l|}{ Produto da concepção* } \\
\hline Nativivo & 324 & 93,9 \\
\hline Natimorto & 18 & 5,2 \\
\hline Aborto & 3 & 0,9 \\
\hline
\end{tabular}

* Os dados são referentes à gestação atual.

gestação em adolescentes (14 a 19 anos) ocorreu em $24 \%$ dos casos $(81 / 337)$. Quanto à procedência, $61,7 \%$ das mulheres eram de Feira de Santana. Mais da metade das mulheres (54\%) referiu ser solteira (182/337), 29,4\% foi o percentual de casadas (99/337); apenas 0,6\% (2/337) eram viúvas. Em 16\% dos prontuários não havia referência ao estado civil. A média de gestações por mulher foi de 2,5 $\pm 2,2 \mathrm{e}$ mediana de 2 (1 a 18). Das 337 gestantes avaliadas, ocorreram 329 gestações únicas $(97,6 \%)$ e 8 gestações duplas $(2,4 \%)$, resultando em 324 nati- 
Tabela 2

Características da assistência pré-natal relacionada à transmissão vertical do HIV e sífilis às 337 gestantes avaliadas em uma Maternidade de Referência. Feira de Santana, Bahia.

\begin{tabular}{lrr}
\hline Características avaliadas & $\mathbf{n}$ & $\%$ \\
\hline Acompanhamento pré-natal (pelo menos uma consulta) & 285 & 84,6 \\
Sim & 28 & 8,3 \\
Não & 24 & 7,1 \\
Não informado & & \\
Fonte de informação sobre pré-natal & 311 & 92,3 \\
Técnico de enfermagem & 62 & 8,4 \\
Enfermeiro & 51 & 15,1 \\
Obstetra & 108 & 32,0 \\
Pediatra & & \\
Sorologia pré-natal para HIV & $24 *$ & 8,4 \\
Referida & 261 & 91,6 \\
Não referida & & 8,0 \\
Sorologia pré-natal para Sífilis (VDRL) & 23 & 92,0 \\
$\quad$ Referida & 262 & \\
Não referida & & \\
\hline
\end{tabular}

* Duas gestantes com sorologia positiva para HIV diagnosticadas no prénatal.

\section{Figura 1}

Proporção de testes rápidos para HIV e VDRL realizados na maternidade, em relação ao acompanhamento pré-natal, nas 313 gestantes que não tinham sido previamente testadas durante o pré-natal, ou para as quais não havia relato de exame. Feira de Santana, Bahia.

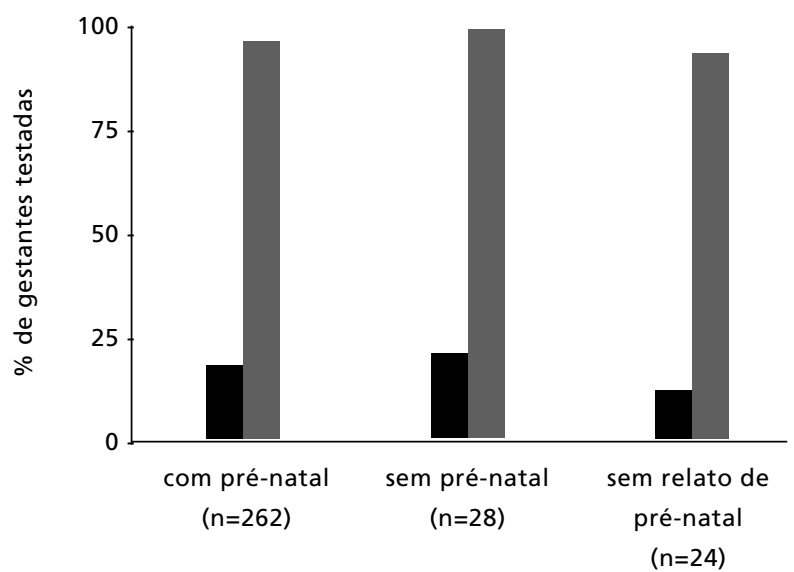

Teste rápido para HIV

VDRL vivos $(93,9 \%), 18$ natimortos $(5,2 \%)$ e 3 abortos $(0,9 \%)$. Quanto à via de parto, 63,8 \% dos partos foram por via vaginal $(215 / 337)$ e $36,2 \%$ foram cesáreas (122/337).

A Tabela 2 ilustra as características da assistência prestada às gestantes em relação ao acompanhamento pré-natal: a maioria das gestantes $(84,6 \%)$ realizou ao menos uma consulta, enquanto $8,3 \%$ não fizeram pré-natal. Em $7,1 \%$ dos prontuários não existiam informações relativas à realização de pré-natal.

O registro de informações sobre realização de pré-natal (sim/não) foi avaliado por categoria profissional. Os técnicos em enfermagem registraram a informação em 92,3\% dos prontuários (311/337), enquanto os enfermeiros do setor referiram em apenas 8,4\% (62/337). Os obstetras (plantonistas ou médicos residentes) referiram a realização ou ausência de pré-natal em $15,1 \%$ dos prontuários (51/337) e os pediatras (preceptores ou médicos residentes) informaram em 32\% dos casos (108/337).

Entre as 285 gestantes que referiram pré-natal, apenas $24(8,4 \%)$ informaram ter feito sorologia para HIV na gestação. Para a maior parte das gestantes que tiveram assistência pré-natal $(91,6 \%)$, não houve solicitação da sorologia ou não havia relato sobre a sua realização em prontuário (261/285). Referente à realização de sorologia para sífilis no pré-natal, $8 \%$ $(23 / 285)$ das gestantes assistidas informaram ter feito o exame na gestação. Para $92 \%$ das gestantes que tiveram assistência pré-natal $(262 / 285)$, não houve solicitação da sorologia ou não havia relato sobre a sua realização em prontuário.

A proporção de gestantes que realizaram o teste rápido para HIV e o VDRL na maternidade, de acordo com o acompanhamento pré-natal, está ilustrado na Figura 1. A análise do grupo das 313 gestantes que não tinham sido previamente testadas durante o pré-natal, ou para as quais não havia relato de exame, mostrou que o teste rápido foi realizado em 56 mulheres $(17,7 \%)$. A proporção de exames realizados na maternidade no grupo de mulheres que foram acompanhadas no pré-natal $(18,4 \%)$ foi semelhante à do grupo sem acompanhamento $(21,4 \%)$. O teste rápido foi rea-lizado na maternidade em $8,3 \%$ das mulheres sem relato de prénatal. Todos os testes rápidos rea-lizados na maternidade foram solicitados pelos enfermeiros do centro obstétrico, não havendo quaisquer referências ao exame nas anotações dos obstetras.

Quanto à triagem para sífilis na maternidade, das 314 gestantes que não tinham sido previamente testadas durante a gestação, ou para as quais não havia relato de exame, o VDRL foi realizado em 306 
Características das gestantes com VDRL reagente, seus recém-nascidos e realização de tratamento para sífilis na maternidade. Feira de Santa, Bahia.

\begin{tabular}{|c|c|c|c|c|c|}
\hline & Caso 1 & Caso 2 & Caso 3 & Caso 4 & Caso 5* \\
\hline \multicolumn{6}{|l|}{ Características maternas } \\
\hline Idade (anos) & 35 & 16 & 29 & 19 & 26 \\
\hline Estado civil & Casada & Solteira & Solteira & Casada & Viúva \\
\hline Procedência & Feira de Santana & Feira de Santana & Outra & Feira de Santana & Feira de Santana \\
\hline \multicolumn{6}{|l|}{ VDRL } \\
\hline Pré-natal & Não informado & Não informado & $1: 2$ & $1: 16$ & Não reagente \\
\hline Maternidade & $1: 16$ & $1: 4$ & $1: 16$ & $1: 16$ & $1: 4$ \\
\hline \multicolumn{6}{|l|}{ Características dos recém-nascidos } \\
\hline Idade gestacional (semanas) & 38 & Não informado & 36 & 39 & 38 \\
\hline Sexo & Masculino & Masculino & Feminino & Feminino & Masculino \\
\hline Peso (gramas) & 3.025 & 2.450 & 2.500 & 3.400 & 2.620 \\
\hline VDRL & Não reagente & Não reagente & $1: 4$ & $1: 2$ & $1: 1$ \\
\hline \multicolumn{6}{|l|}{ Tratamento da sífilis } \\
\hline Da gestante & Não & Não & $\operatorname{Sim}$ & Não & Não \\
\hline Do recém-nascido & Não & Não & $\operatorname{sim}$ & Sim & Não \\
\hline
\end{tabular}

* Caso 5: co-infecção HIV/sífilis.

mulheres $(97,5 \%)$. A proporção de exames realizados no grupo de mulheres acompanhadas no prénatal foi de $97,3 \%$. O VDRL foi realizado em $100 \%$ das gestantes sem acompanhamento pré-natal e em $95,8 \%$ das gestantes sem referência a acompanhamento na gestação. Além disso, apenas uma entre as 23 mulheres que fizeram VDRL no pré-natal não repetiu o exame na maternidade.

Foram identificadas duas gestantes infectadas pelo HIV, diagnosticadas no pré-natal. Os partos ocorreram a termo, um por cesárea e outro por via vaginal; ambos foram do sexo masculino. Não houve recém-nascido com baixo peso ao nascer. As membranas amnióticas estavam íntegras nos dois casos. Em ambos, a profilaxia anti-retroviral da transmissão vertical (uso de AZT) foi iniciada na gestação e continuada na maternidade.

As características das cinco gestantes com VDRL reagente e dos seus recém-nascidos estão descritas na Tabela 3: a idade materna variou entre 16 e 35 anos (média $25 \pm 7,7$ ). Em relação ao estado civil, $40 \%$ eram casadas $(2 / 5), 40 \%$ solteiras $(2 / 5) \mathrm{e}$ $1 / 5$ declarou-se viúva (20\%). Para $80 \%$ das mulheres com VDRL reagente $(4 / 5)$ não havia referência a tratamento na maternidade. Uma gestante (caso 3 ) informou tratamento três anos antes da gestação atual, apresentando aumento de título do VDRL em relação ao exame pré-natal, sendo iniciado tratamento na maternidade. A gestante referida como "caso 4" concluiu o tratamento na semana anterior ao parto. Não houve relato de realização de teste confirmatório para as gestantes que apresentaram VDRL reagente.

Em relação aos recém-nascidos, ocorreu um caso de prematuridade (idade gestacional menor que 37 semanas), representando $20 \%$ (1/5); a razão de sexos foi $3: 2$, predominando o masculino. Apenas um recém-nascido $(20 \%)$ apresentou baixo peso ao nascer (peso menor que $2.500 \mathrm{~g}$ ), com idade gestacional não informada no prontuário. Dois recémnascidos tiveram diagnóstico de sífilis congênita e receberam tratamento; ambos eram assintomáticos. Outro recém-nascido apresentou VDRL reagente $1: 1$, sendo VDRL materno reagente (1:4) e não trazia informações nos prontuários obstétrico e pediátrico sobre investigação diagnóstica e/ou tratamento.

\section{Discussão}

A avaliação do primeiro ano de implantação do "Projeto Nascer Maternidades" na Instituição onde foi realizado o estudo demonstrou que a triagem para HIV na maternidade foi pequena, sendo realizado teste rápido apenas para $17,7 \%$ das gestantes que desconheciam seu estado sorológico. Contrariamente, a quase totalidade das gestantes admitidas realizou VDRL na maternidade, aproximando-se da 
meta do "Projeto Nascer-Maternidades" de avaliar todas as gestantes. A elevada realização do VDRL pode ser explicada pelo fato de a alta hospitalar nessa maternidade estar atrelada ao resultado negativo do VDRL do binômio mãe-filho, o que não ocorre em relação ao HIV. Além disso, a triagem para sífilis é uma rotina implantada na instituição há quase duas décadas, enquanto a triagem para HIV é mais recente.

Referente à profilaxia da transmissão vertical do HIV, os resultados obtidos nesse estudo foram inferiores àqueles encontrados em Vitória da Conquista, outra cidade de médio porte do Estado da Bahia, onde o impacto do "Projeto Nascer Maternidades" (implantado em 2001) pode ser medido pelo incremento na realização de teste rápido para HIV nas maternidades: de $276 \%$ em 2002 e de $170 \%$ em 2003.18

$\mathrm{O}$ pequeno número de testes rápidos para HIV realizados na maternidade do estudo no período avaliado não poderia ser atribuído à falta de insumos, uma vez que o teste faltou apenas durante o período de 17 de maio a 7 de junho de 2004, o que envolveu 17 gestantes (menos de 5\% da nossa amostra). A pequena participação $(33,3 \%)$ dos médicos obstetras da instituição nas oficinas de capacitação para o "Projeto Nascer Maternidades" poderia ter um papel importante. Esse fato pode explicar a razão pela qual não havia nenhuma referência médica nos prontuários obstétricos a respeito de solicitação e resultados desses exames, sendo a totalidade dos testes rápidos solicitada pelos enfermeiros do setor. Outros fatores foram ainda identificados, como a escala de trabalho dos enfermeiros do centro obstétrico, compartilhada por outros setores durante um mesmo turno de trabalho, com instalações afastadas do centro obstétrico, gerando uma fragmentação da assistência. A inexistência de neonatologista em sala de parto na instituição dificulta a integração entre a obstetrícia e a neonatologia, imprescindível para a adequada assistência perinatal e aplicação do protocolo em questão.

O acompanhamento pré-natal (ao menos uma consulta) foi realizado para $84,6 \%$ das mulheres da amostra, concordando com os dados do Ministério da Saúde (85\%). ${ }^{19}$ Porém, não estavam disponíveis informações que pudessem subsidiar uma avaliação qualitativa. Entretanto, a ausência de referência da solicitação de sorologia anti-HIV para a maioria das gestantes durante o pré-natal, poderia constituir um marcador de pouca qualidade da assistência, além de dificultar a profilaxia da transmissão vertical na maternidade. Esses dados são preocupantes, uma vez que havia indicação precisa para triagem sorológica de 313 mulheres na maternidade e apenas 56 testes foram realizados $(17,7 \%$ dos indicados $)$. Essa situação configura-se em oportunidade perdida de identificação de gestantes elegíveis para profilaxia da transmissão vertical, o que pode representar para os filhos dessas mulheres uma restrição do direito de nascer e viver sem Aids.

Quanto à terapêutica, foi aplicada adequadamente para os filhos das gestantes com diagnóstico de sífilis, entretanto, proporcionou tratamento inicial para apenas $20 \%$ das mães, sem referência a tratamento de seguimento. Nenhuma parturiente com VDRL reagente foi testada para HIV na maternidade, apesar da recomendação de investigar a coinfecção20 devido à alta prevalência de sífilis entre os soropositivos para HIV e interferência no prognóstico das duas doenças. 21,22 Também não havia nos prontuários quaisquer referências à orientação de tratamento ambulatorial e à indicação de tratamento dos parceiros sexuais.

Em relação às gestantes infectadas pelo HIV, uma teve parto cesárea e a outra, parto vaginal. Ambas estavam com 38 semanas de gestação e membranas amnióticas íntegras. Embora a dilatação cervical e a carga viral materna estejam implicadas na indicação da via de parto15,23-27 não existiam nos prontuários dessas gestantes quaisquer informações referentes a estas variáveis e à opção pela via de parto.

Alguns obstáculos foram encontrados durante a coleta de dados, merecendo destaque a insuficiência de informações em prontuários, requerendo busca de outras fontes de dados.

Esse estudo demonstrou que a implantação do Projeto Nascer-Maternidades no primeiro ano na maternidade avaliada não trouxe resultados efetivos, traduzidos pela baixa solicitação de teste rápido para HIV para gestantes com sorologia desconhecida e ausência de orientação terapêutica adequada para as parturientes com sífilis.

Pelo exposto, a sensibilização e capacitação da equipe de assistência perinatal devem ser contínuas, além da avaliação sistemática do "Projeto Nascer Maternidades", consolidando o seguimento das 
normas. Dessa forma, a meta do Ministério da Saúde para redução da transmissão vertical do HIV e da sífilis poderá ser alcançada.

\section{Referências}

1. Brasil. Ministério da Saúde. Coordenação Nacional de DST/HIV/AIDS. Unidade de Informação e Vigilância (UIV). Dados epidemiológicos AIDS Tabela VII. Bol Epidemiol-Aids e DST. 2004; Ano I (1: 1 $1^{\mathrm{a}}-26^{\mathrm{a}}$ semanas). (periódico online). [Acesso em: 12 fev. 2006]. Disponível em: http://www.aids.gov.br/data/documents/stored Documents/\%7BB8EF5DAF-23AE-4891-AD36-1903553A 3174\%7D/\% 7B47CC4C73-91C6-4E44-A670-3D92ADF2 A59E\%7D/BOLETIM2.pdf

2. Brasil. Ministério da Saúde. Secretaria de Vigilância em Saúde. Sistema Nacional de Vigilância em Saúde. Relatório de situação: Bahia. 2006. 2. ed. 8 p. [acesso em: 30 jul. 2006]. Disponível em: http://portal.saude.gov.br/portal/ arquivos/pdf/relatorio_snvs_ba_2ed.pdf

3. Guinsburg R. Sífilis congênita. In: Farhat CK, organizador. Infectologia pediátrica. 2. ed. São Paulo: Atheneu; 1998. p. 323-34.

4. Brasil. Ministério da Saúde. Secretaria de Vigilância em Saúde. Programa Nacional de DST e Aids. Diretrizes para o Controle da Sífilis Congênita. Brasília, DF; 2005. p. 44-54. (Série Manuais n. 62).

5. Connor EM, Sperling RS, Gelber R, Kiselev P, Scott G, O'Sullivan MJ, VanDyke R, Bey M, Shearer W, Jacobson RL, Jimenez E, O'Neill E, Bazin B, Delfraissy JF, Culnane M, Coombs R, Elkins M, Moye J, Stratton P, Balsley J, for The Pediatric AIDS Clinical Trials Group Protocol 076 Study Group. Reduction of maternal-infant transmission of human immunodeficiency virus type 1 with zidovudine treatment. N Engl J Med. 1994, 331: 1173-80.

6. Shaffer N, Chuachoowong R, Mock PA, Bhadrakom C, Siriwasin W, Bangkok Collaborative Perinatal HIV Transmission Study Group. Short-course zidovudine for perinatal HIV-1 transmission in Bangkok, Thailand: a randomised controlled trial. Lancet. 1999; 353: 773-80.

7. Bitnun A, King SM, Arneson C, Read SE. Failure to prevent perinatal HIV infection. Can Med Assoc J. 2002; 66: 904-5.

8. Marques HHS, Latorre MRDO. Falhas na identificação da infecção pelo HIV durante a gravidez em São Paulo, 1998. Rev Saúde Pública. 2002; 36: 385-92.

9. Tess BH, Rodrigues LC, Newell ML, Dunn DT, Lago TD, São Paulo Collaborative Study for Vertical Transmission of HIV-1. AIDS-breastfeeding, genetic, obstetric and other risk factors associated with mother-to-child transmission of HIV-1 in São Paulo State, Brazil. J Int AIDS Soc. 1998; 12: 513-20.

10. Succi RCM, Grupo de Estudo da Sociedade Brasileira de Pediatria. Transmissão vertical do HIV no Brasil em 20032004: resultado preliminar de um estudo colaborativo multicêntrico. J Paranaense Pediatr. 2005; 6 (1) [periódico online]. [acesso em: 24 abr. 2006]. Disponível em http://157.86.8.37/docs/taxa\%20de\%20transmissao\%20vert ical.pdf
11. Brasil. Ministério da Saúde. Assistência pré-natal: normas e manuais técnicos. 3 ed. Brasília, DF; 1998.

12. Gust DA, Levine WC, Saint Louis ME, Braxton J, Berman SM. Mortality associated with congenital syphilis in the United States, 1992-1998. Pediatrics. 2002; 109: 79-89.

13. Southwick KL, Guidry HM. An epidemic of congenital syphilis in Jefferson County, Texas, 1994-1995: inadequate prenatal syphilis testing after an outbreak in adults. Am J Public Health. 1999; 89: 557-60.

14. Brasil. Ministério da Saúde. Portaria n ${ }^{\circ} 2.104$ de 19 de novembro de 2002. Institui no âmbito do Sistema Único de Saúde - SUS o Projeto Nascer-Maternidades. Diário Oficial da União (DOU). Brasília, DF; nov. 2002, Seção I, p. 48.

15. Brasil. Ministério da Saúde. Secretaria de Políticas de Saúde. Coordenação Nacional de DST/HIV/AIDS, Áreas Técnicas de Saúde da Mulher e Nutrição. Manual para Oficina de Capacitação Projeto Nascer-Maternidades. Brasília, DF; 2002.

16. Brasília. Ministério da Saúde. SINAN (Sistema Nacional de Agravos de Notificação) Brasil. [base de dados] [acesso em: 14 nov. 2006]. Disponível em: http://portal.saude. gov.br/portal/arquivos/pdf/relatorio_snvs_ba_2ed.pdf.

17. IBGE (Fundação Instituto Brasileiro de Geografia e Estatística). Banco de dados: cidades [base de dados]. [acesso em: 19 jan. 2005]. Disponível em: http://www. ibge.gov.br

18. Brasil. Ministério da Saúde. Programa Nacional de DST/HIV/AIDS. Avaliação das oficinas do Projeto NascerMaternidades 2004. [monografia online] [acesso em: 19 maio 2004]. Disponível em: http: //www.aids.gov.br/ final/tratamento/politicas/avaliacao_projeto_nascer.htm

19. Brasil. Ministério da Saúde. Secretaria de Vigilância em Saúde. Departamento de Análise de Situação de Saúde Saúde Brasil 2004 - uma análise da situação de saúde. Brasília, DF; 2004. (Série G: Estatística e Informação em Saúde).

20. Schultze GE, Landers S. Management of infants born to women with sexually transmitted diseases. Am Fam Phys. 1994; 50: 1479-86.

21. Buntin DM. The 1993 sexually transmitted disease treatment guidelines. Sem Dermatol. 1994; 13: 269-74.

22. Hutchinson CM, Hook III EW, Shepherd M, Verley J, Rompalo AM. Altered clinical presentation of early syphilis in patients with human immunodeficiency virus infection. Ann Intern Med. 1994, 121: 94-100.

23. Dunn DT, Newell ML, Mayaux MJ, Kind C, Hutto C, Goedert JJ, Andiman W. Mode of delivery and vertical transmission of HIV-1: a review of prospective studies. Perinatal AIDS Collaborative Transmission Studies. J Acquir Immune Defic Syndr. 1994; 7: 1064-6. 
24. Garcia PM, Kalish LA, Pitt J, Minkoff H, Quinn TC, Burchett SK, Kornegay J, Jackson B, Moye J, Hanson C, Zorrilla C, Lew JF. Women and Infants Transmission Study Group. Maternal levels of plasma human immunodeficiency virus type 1 RNA and the risk of perinatal transmission. N Engl J Med. 1999; 341: 394-402.

25. Hudson CN. Elective caesarean section for prevention of vertical transmission of HIV-1 infection. Lancet. 1999; 353: 1030-1.

Submetido em 11 de fevereiro de 2008

Versão apresentada em 25 de setembro de 2008

Aprovado em 3 de outubro de 2008
26. Landesman SH, Kalish LA, Burns DN, Minkoff H, Fox HE, Zorrilla C, Garcia P, Fowler MG, Mofenson L, Tuomala R. Obstetrical factors and the transmission of human immunodeficiency virus type 1 from mother to child. The Women and Infants Transmission Study. N Engl J Med. 1996; 334: 1617-23.

27. Minkoff H. Prevention of mother-to-child transmission of HIV. Clin Obst Gynecol. 2001; 44: 210-25. 